\title{
Exploration and Analysis on Incidences of Covid-19 on the Senior Peoples and TCM Management-60 Clinical Cases
}

\author{
Dan Jiang MMedSc TCM \\ Visiting professor (Beijing University of Chinese Medicine China), Fellow of British Acupuncture Council (BAcC), Fellow of Association of \\ Traditional Chinese Medicine (ATCM).
}

*Corresponding Author: Dan Jiang, Visiting professor (Beijing University of Chinese Medicine China), Fellow of British Acupuncture Council (BAcC), Fellow of Association of Traditional Chinese Medicine (ATCM).

Received date: June 17, 2021; Accepted date: June 25, 2021; Published date: June 29, 2021

Citation: D Jiang. (2021) Exploration and Analysis on Incidences of Covid-19 on the Senior Peoples and TCM Management-60 Clinical Cases. Journal of Clinical and Laboratory Research. 3(1); DOI:10.31579/2768-0487/033

Copyright: (C2021 Dan Jiang. This is an open-access article distributed under the terms of the Creative Commons Attribution License, which permits unrestricted use, distribution, and reproduction in any medium, provided the original author and source are credited.

\begin{abstract}
:
Incidence and prevalence of infected Covid-19 are still rampant in the West and the most countries throughout world from 2020 to 2021. Although the incidence of infected Covid-19 in the developed countries has decreased down by the popular vaccination, it is still anxiety and worry to make more serious widespread from the variated coronavirus. It is a rigorous fact that there still are higher on the mortality to the seniors, special seniors who are resided at nursing homes. For seniors, the infected Coronavirus may product them a disastrous attack according to their pre-existing diseases, complication accompanied with Covid-19, and post-syndromes caused by Covid-19. Their Covid-19 may be more complex, more serious, harder to find a fast and effective therapies and easier to become to the post-syndromes which will make a bad influence to the quality of life in the rest of their life.

TCM focuses to individual case, and pay attention more for their pre-existing diseases, complication with Covid19, and possibility of post-syndrome's production while some anti-coronavirus herbs are prescribed, so a more fixed prescription is made. In this clinical trial, the author classified 60 cases into two groups: over 45 years old as the observing group and under of 44 years old as the contrasted group, incidences on pre-existing diseases, complication and post-syndromes are observed separately; their results after treated by Chinese medical herbs are also observed. We recognize from these observation and analysis TCM treatment can significantly stabilized pre-existing diseases, treat complication together and prevent the post-syndromes' occurrence while is treating to Coronavirus. TCM is a suitable treating choice to Senior with Covid-19.
\end{abstract}

Key words: seniors; Covid-19; post-syndrome of Covid-19; TCM

\section{Introduction:}

Incidence and prevalence of infected Covid-19 are still rampant in the West and the most countries throughout world from 2020 to 2021 [1]. Although the incidence of infected Covid-19 in the developed countries has decreased down by the popular vaccination, it is still anxiety and worry to make more serious widespread from the variated coronavirus [2]. It is a rigorous fact that there still are higher on the mortality to the seniors, special seniors who are resided at nursing homes. For seniors, the infected Coronavirus may product them a disastrous attack according to their pre-existing diseases, complication accompanied with Covid-19, and post-syndromes caused by Covid-19. Their Covid-19 may be more complex, more serious, harder to find a fast and effective therapies and easier to become to the post-syndromes which will make a bad influence to the quality of life in the rest of their life.

Background:

From daily report issued by WHO in the edition 43 in 8th Jun 2021:
More than 100 million of confirmed cases $(172,637$ 097) in the world;

More than 3 million of deaths $(3,718,944)$ in the world

More than 3 million of new cases in last 7 days $(3,016,005)$ in the world.

\section{From the daily report issued by GOV. UK}

More than 4 million of confirmed cases in UK $(4,550,944)$

More than 120 thousands of deaths in UK $(127,884)$

Traditional Chinese medicine (TCM) actually is an effective therapy which can not only treat infected coronavirus, can but also control coronavirus prevalence and prevent its development. TCM which was overall used in the temporary hospitals (Fangchang hospitals) in where, more than $90 \%$ of patients treated with Chinese herbal medicine who did not progress from the minor or milder stages of the disease to the severe or critical stage. In all of them the positive PCR became negative; herbs 
were involved in the rescue and treatment of severe and critical cases in ICU of hospitals as well. So it is an important factor in how Covid-19 was controlled so quickly in China.

In the West, it may be from a political reason, possible influenced by a fixed impression with pride and prejudice, the social works, scientific and medical professions have a serious insufficiency to recognize the antiCovid-19 effect of TCM. As a TCM consultant, we are not satisfactory to only treat these patients with Covid-19, but we should report our clinical cases who can make the herbal effect in the UK and demonstrate the treating superiority from TCM here.

\section{Methods :}

In this clinical trial, I collect 60 cases who I have treated and remained full of clinical reports from April 2020 to March 2021; I classified these cases into two groups: over 45 years old as the observing group and under of 44 years old as the contrasted group. The incidences on pre-existing diseases, complication and post-syndromes are observed separately on both of groups; their results after treated by Chinese medical herbs are also observed.

Among of patients who we have been treating:

$$
>45 \text { year olds as the observation group is } 27 \text { cases; }
$$

$<44$ year olds as contrasted group is 33 cases.

\section{Observation:}

\section{Incident rate of Covid-19:}

Infectious symptoms with a positive PCR : 29例

Suspected cases without a positive PCR : 31例*

Gentle stage; minor clinical symptoms, $\mathrm{T}<38$

Ordinary stage: $\mathrm{T}>38.1$, typical symptoms

Severe stage or more severe: $\mathrm{T}>39$, Breathless with dyspnea, or other severe symptoms.

\begin{tabular}{|c|c|c|}
\hline Diseased levels & $\begin{array}{c}\text { Over than 45 years old } \\
\text { group (27) }\end{array}$ & $\begin{array}{c}\text { Under of 44 years old } \\
\text { group (33) }\end{array}$ \\
\hline Gentle Stage & $12(44 \%)$ & $11(33 \%)$ \\
\hline Ordinary Stage & $11(41 \%)$ & $22(67 \%)$ \\
\hline Severe and over more & $4(15 \%)$ & $33(55 \%)$ \\
\hline All & $27(45 \%)$ & \\
\hline
\end{tabular}

Table 1: Incidences and diseased levels on Patients in different years old groups with Covid-19.

*Note: in the early phase of pandemic Covid-19, the PCR test was not convenient, so many patients who couldn't make their test, or disliked to be tested although they manifested many symptoms can be diagnosed in the clinic as a suspected cases.

\section{Incident rates of Special relevant health problems:}

2.1 Pre-existing diseases which the patients suffered before got their covid-19: such as Psychtric disorders, Diabetes, IBS etc.

\begin{tabular}{|c|c|c|}
\hline the Basic diseases & $\begin{array}{c}\text { Over than 45 years old } \\
\text { group (27) }\end{array}$ & $\begin{array}{c}\text { Under of 44 years old } \\
\text { group (33) }\end{array}$ \\
\hline Irritable bowel syndrome & 3 & 2 \\
\hline Cardiologic vascular disease & 2 & \\
\hline Endometriosis / Functional uterus bleeding & 2 & \\
\hline PCO/ PCOS & 1 & \\
\hline Allergy & 5 & \\
\hline Asthma / Respiratory diseases & 1 & \\
\hline Transient Ischaemic Attack & 5 & \\
\hline Gastritis / Colitis & 8 & \\
\hline Arthritis / Joints pain & 1 & \\
\hline Acute / Chronic Eczema & 3 & \\
\hline Post-virus, Chronic Fatigue Syndrome & 1 & \\
\hline Post-cancers & $32(119 \%) *$ & \\
\hline All & & \\
\hline
\end{tabular}

Table 2: Compare on incidence of the pre-existing diseases with Covid-19 on groups over 45 and under of 44.

*One patient can suffer from more than a disease, so

The group with over 45 years old: 33 diseases in 27 cases, which is engaged as $119 \%$;

The group with under 44 years old: 6 diseases in 33 cases which is engaged as $18 \%$.

2.2 Complication: the patients suffered during the Covid-19 is infected or related with it: Such as Lung collapse, Cardiological problem, Reproductive disorders etc. 


\begin{tabular}{|c|c|c|}
\hline The Complication & $\begin{array}{c}\text { Over 45 years old group } \\
(\mathbf{2 7})\end{array}$ & $\begin{array}{c}\text { Under of 44 years old group } \\
\mathbf{( 3 3 )}\end{array}$ \\
\hline Irritable bowel syndrome & 5 & 1 \\
\hline Colitis & 1 & \\
\hline Psychiatric disorders & 4 & 1 \\
\hline Reproductive disorders & 5 & 2 \\
\hline Lung weaker & $15(56 \%)$ & $4(12 \%)$ \\
\hline All & & \\
\hline
\end{tabular}

Table 3: Compare on incidence of Complication during Covid-19 with groups over 45 and under of 44.

Among of the group with over 45 years old: 15 cases with complications, $56 \%$;

Among of the group with under 44 years old: 4 cases with complications, $12 \%$.
2.3 Post-syndrome of Covid-19: some clinical symptoms are still existing after PCR become negative; Such as lung failure, Heart failure, Psychtric disorders, Indigestive problem etc.

\begin{tabular}{|c|c|c|}
\hline $\begin{array}{c}\text { Post-syndrome of Covid-19 } \\
\begin{array}{c}\text { Lung weaker/ Respiratory function } \\
\text { failure / Lung Fibrosis }\end{array}\end{array}$ & $\begin{array}{c}\text { Over than 45 years } \\
\text { old group (27) } \\
\text { group (33) }\end{array}$ \\
\hline Heart failure & 5 & 2 \\
\hline Colitis / IBS & 1 & 1 \\
\hline Psychiatric disorders & 3 & 2 \\
\hline Post-virus, chronic-fatigue syndrome & 2 & 2 \\
\hline All & $14(51 \%)$ & $7(21 \%)$ \\
\hline
\end{tabular}

Table 4: Compare on incidence of Post-syndrome of Covid-19 with group over 45 and under of 44.

Among of the group with over 45 years old: 14 cases who become to post syndrome of Covid-19, 51\%;

Among of the group with under 44 years old: 7 cases who become to postsyndrome of Covid-19, $21 \%$.

\section{TCM managements}

\subsection{Special diagnosis:}

We diagnose the infected or suspected Covid-19, according to these diagnostic methods:

- Clinical symptoms: 60 cases;

- Tongue appearances: 59 cases (a baby in 11 months was ignored on his tongue.)

- PCR: 29 cases with positive result who were diagnosed as infected Covid-19;

31 cases who did not test, or disliked to be test for PCR were diagnosed as suspected Covid-19

- $\quad$ Other diagnostic methods, such as $\mathrm{SaO} 2, \mathrm{X}$-ray, MRI etc. 5 cases.

\subsection{TCM management:}

Due to our using remote diagnosis with an indirect contact access, so I prefer to prescribe herbal medicines in different forms according to the severity of disease and patient's preference to individual case, I prescribed herbal treating form as below:

- Special prescribed raw herbs in a decoction form to individual case: 37 cases;
- Special prescribed concentrated herbal powder form to individual case: 6 cases;

- $\quad$ Prepared herbal powders or pills which I designed for common conditions to prevent or treat Covid-19 in advance: 56 cases.

Because prepared herbal sachets or pills are cheaper and easily take, so many patients can be prescribed raw herbs or powder herbs at the early stage who manifested as a severe or typical symptoms as Covid-19, then they were transferred to a prepared herbal pills or sachets after they had been better in 1-3 weeks.

\section{- Acupuncture:}

I can do acupuncture treatment after we were reopening the clinic, so I select acupuncture with herbs together for making a stronger enough treating level. The most of patients, special these ones with psychtric symptoms, or complex state, or complication, or postsyndromes, acupuncture will play a necessary and valuable effect.

\subsection{Routine Treatment of TCM}

* Anti-Coronavirus Treatment with herbal medicine : I will select the 12 main formula as the basic prescription as below, then I will variate with some herbs according to special symptoms which patient manifests for making a complete prescription to individual case.

Huoxiangzhengqi Decoction / Pills ;

Huopoxialing / Lianpo Decoctions;

Sanren Decoction;

Above three formulas should be selected to infectious Covid-19 patients in an early stage or gentle cases: Huoxiangzhengqi Decoction is better to 
be given to the patients with wind-cold-damp pathogenic factors; Huopoxialing / Lianpo Decoctions is better to be given to the patients with wind-heat-damp pathogenic factors; Sanren Decoction is better to be given to the patients with dampness.

Lianhuaqingwen Cap / Huolingshuanghua Yin;

Qingfeipaidu Decoction;

Above three formula should be selected to infectious Covid-19 patients in an ordinary stage, or middle or severe cases. Qingfeipaidu Decoction is a basic main prescription; Lianhuaqingwen Cap / Huolingshuanghua Yin are prepared herbal capsule and granule.

Special prescription which is variated with above basic formulas to individual cases according to his/her basic health state, complication and possible post-syndromes.

\section{Clinical summary:}

\subsection{Treating result on 60 cases:}

Criterion: 1) Cured: all of clinical symptoms disappeared;

PCR become negative;

Pre-existing diseases are stables;

No complication appeared;

No post-syndromes appeared.

2) Better: PCR become a negative; Symptoms get less;

Post-syndrome is still existing.

Result:

\begin{tabular}{|c|c|c|}
\hline Result & $\begin{array}{c}\text { Over 45 years old } \\
\text { group (27) }\end{array}$ & $\begin{array}{c}\text { Under of 44 years } \\
\text { old group (33) }\end{array}$ \\
\hline Cured & $13(48 \%)$ & $26 \quad(79 \%)$ \\
\hline Better & $14(52 \%)$ & $7 \quad(21 \%)$ \\
\hline
\end{tabular}

Table 5: Compare on Result of TCM treatment to Covid-19 in groups over 45 and under of 44.

\subsection{Cases study:}

Case 1: a typical sufferer with Covid-19 over stress and multiple pre-existing illnesses:

Ms. T, 56 Barrister with stress, irritable bowel and shoulder-neck pain who was affected Covid-19 from an excited kiss gave by one of her client when she won the lawsuit for her fostering right to son. Due to a busy work, she has been manifesting stress, restless, nervous, headache, poor sleep and multiple joint pains last a couple of years, she was caught up a Coronavirus immediately after she attended her court.

Main complaint: fever T 38.4c, fatigue, aching in general, illness feeling, some cough without phlegm, restlessness and hard fail asleep. PCR is positive.

TCM signs: light red tongue with greasy coating

Diagnosis: 1) Infectious Covid-19

2) Multiple pre-existed diseases: Stress, Psychtric disorder-Anxiety, Irritable bowel syndrome (IBS), Arthritis

Differentiation of syndrome: Dampness and heat accumulated at liver \& spleen
Herbal prescription: Huoxiangzhengqi variated with Xiaochaihu Decoction

Huoxiang10, Houpo10, Jiangbanxia10, Fuling10,

Huashi15, Yiyiren30, Chaihu10, Chishao10,

Huangqin10, Yuxingcao10, Zhuye10, Gancao5.

Herbal pills: Qianghuoshengshi Pills

Huoxiangzhengqi Pills

Result: Due to both of treating principles on anti-coronavirus and releasing stress together are given, she is healed and relaxed quickly.

\section{Case 2: a case who suffers from three times of Covid-19 is complicated with Colitis:}

Ms. K, 55 teacher who was suffered from a severe Ulcerative Colitis was controlled well by medicine and TCM's support 2 years ago. Since 2020, she was suffered from Covid-19 for 3 times in Feb, Aug and Jan 2021, she was also triggered to her Colitis; since she is third infected by Coronavirus, she is aggravated her colitis until PCR become negative.

Complaints: diarrhea with watery stools countless daily, more than 10 times with abdominal cramps, but no blood or mucus in stools; fatigue with general aching and poor sleep.

TCM signs: pale tongue with thin coating

Diagnosis: 1) Infectious Covid-19

\section{2) Complication-Ulcerative Colitis;}

Differentiation of TCM: Excessive damp and heat accumulation with spleen deficiency.

Herbal prescription: Baitouwong30, Qinpi10, Baishao20, Yanhusuo10, Ganjiang10, Chenpi10, Baizhu10, Fangfeng10,

Kezi10, Yizhiren10, Huangbai10, Zhimu10

Gegen10, Zhigancao5

Result: after she takes the special prescription, her Covid-19 is completely healed and diarrhea and abdominal cramp caused by colitis is also gradually controlled well. Due to Buzhongyiqi decoction is replaced after her diarrhea is controlled, she has been getting stabilization in general.

\section{Case 3: a case with lung weaker as a post syndrome after} be controlled from Covid-19:

Ms. N 67 therapist, due to infected Covid-19 two months ago, she had fever, 39.6c, cough, chest pain and dyspnea, SoA2 <90, so she was admitted into hospital for intensive treatment for a week. After discharged, she is still feel lethargy, breathless, tiredness in general. If move, she can cough or breathlessness, thirsty, poor sleep. After a course of antibiotics, she doesn't feel better at all.

TCM signs: light red tongue with less white coating;

Test signs: PCR -, SoA2 92-95 swings

Diagnosis: 1) Post-syndrome of Covid-19

2) Lung weaker / Respiratory functional Insufficiency

Differentiation of TCM: lung and spleen Qi and Yin deficiency

Herbal prescription:

Taizishen30, Nan / Beishashen15 each, Maimendong10, Shendihuang10, Tinglizi10, Jiegeng10, Banzhilian10, Yuxingcao10, 
Sangbaipi10, Huangqin10, Zhimu10, Gancao5.

Acupuncture: Moxi at Ren8,

Du20, 24 St8, 25, 36, Ren 15, 12, 6, 4, Sp9, 6, 4, 3 Sj5, Gb41

Result: She have been controlled for all of symptoms and weakness lungs after 4 weeks of TCM managements.

\section{Discussion :}

$1 \mathrm{TCM}$ is a good choice to over 45 senior group; the main symptoms which are caused from Covid-19 can be controlled after effective antivirus treatment.

2 Due to TCM can manage a formula targeting to individual case, his/her pre-existing diseases can be involved or double cared in the prescription, so a stable state on their pre-existing diseases can be received after the main symptoms from Covid-19 is treated;

3 Due to TCM see a body as a whole, so their complications accompany with Covid-19 can also be paid attention more for healed together.

4 TCM can effectively prevent and treat post-syndromes of Covid-19. Due to TCM can kill Coronavirus, stabilize immune system, promote organ's function and correct bio-circulation, so it can make a complete rehabilitation after the Covid-19 Is controlled.
Due to stabilize pre-existing diseases, treat complications and preventing post-syndromes accompanied with anti-coronavirus with TCM, so TCM can display a significant comprehensive treating effect to the senior people.

\section{Conclusion :}

TCM is an effective and suitable treating choice for over 45 senior peoples with Covid-19.

\section{Reference:}

1. Internet: World Health Organization. (2021) Weekly operational update on Coronavirus disease 2019 (COVID-19).

2. Internet: Covid-19 daily report, GOV.UK (2021).

3. National Health Commission \& National Administration of TCM. (2020) Diagnosis and Treatment Protocol for Novel Coronavirus Pneumonia - Trial Version.

4. D Jiang. (2021) Diagnosis and Management of Infected or Suspected Patients with Covid-19 A study of 48 Cases in the UK, European Journal of Oriental Medicine, Iss P.

5. D Jiang. (2021) Guidelines for Patients-practitioner Contact and TCM Management in Post- syndromes of Covid-19 the Journal of Chinese Medicine. Iss125, P71-79.

6. Z Zheng, D Jiang etc. Efficacy of Traditional Chinese Medicine on Covid-19: Two Case Reports Medical acupuncture Vol33, No1 P92-102.
This work is licensed under Creative Commons Attribution 4.0 License

\section{To Submit Your Article Click Here: Submit Manuscript}

DOI: $10.31579 / 2768-0487 / 033$
Ready to submit your research? Choose Auctores and benefit from:

* fast, convenient online submission

* rigorous peer review by experienced research in your field

* rapid publication on acceptance

* authors retain copyrights

* unique DOI for all articles

* immediate, unrestricted online access

At Auctores, research is always in progress.

Learn more www.auctoresonline.org/journals/journal-of-clinical-andlaboratory-research 